\title{
Induction of odor preference in a specialist insect parasitoid
}

\author{
LAURE KAISER and RUURD DE JONG \\ Laboratoire de Neurobiologie Comparée des Invertébrés, INRA-CNRS, Bures-sur-Yvette, France
}

\begin{abstract}
This paper deals with the effect of associative and nonassociative odor conditioning on odor preference in a specialist larval parasitic wasp of Drosophila melanogaster, Leptopilina boulardi. Differently experienced females were offered a choice between banana and strawberry odors in a fourarmed olfactometer. Wasps that were allowed to oviposit in an odorless airflow before testing exhibited no significant response to the odors, whereas females with an oviposition experience in the presence of an odor clearly preferred the scent that they had experienced. Furthermore, exposure to strawberry odor after oviposition could also induce a preference for strawberry or banana odor, depending on the concentrations of both scents used for conditioning and testing. This effect was independent of the time elapsed between oviposition and strawberry exposure, suggesting that it was caused by nonassociative conditioning and not by backward associative learning. Exposure to banana odor after oviposition had no effect on females' responses. Variations in odor concentrations also influenced the expression of associative learning, although to a much lesser extent.
\end{abstract}

Odors are major signals used by many parasitoid insects to locate suitable hosts. An increase in behavioral responses to volatiles that have been detected during parasitization seems to be a widespread phenomenon. Associative learning when an odor and an oviposition experience are associated is thought to be the main mechanism involved (see, e.g., De Jong \& Kaiser, 1991; Lewis \& Tumlinson, 1988; Vet \& Groenewold, 1990; see also Turlings, Wäckers, Vet, Lewis, \& Tumlinson, 1993, for a review). Odor learning can change odor preferences and is hypothesized to be advantageous to parasitoid species searching for hosts in a variety of habitats. A well-studied example is the generalist Leptopilina heterotoma. It parasitizes a variety of Drosophila species living in different habitats, such as fermenting material, decaying mushrooms, and decaying plant material. Oviposition in one of these habitats can change the innate preference for host-habitat odors (Vet, Lewis, Papaj, \& Van Lenteren, 1990).

The present study deals with the effects of odor learning on subsequent odor preference in a specialist parasitoid of Drosophila larvae, Leptopilina boulardi Barb.

The authors are very grateful to Minh-Hà Pham-Delègue, Yves Carton, and Louise Vet for discussion and comment; two anbnymous reviewers for their constructive criticisms; Yves Carton for providing the insect strains; Françoise Frey and Chérif Guenfoud for their help with the insect rearing; Heather McAuslane for linguistic advice; and Mr. Delache and Laurence Pinczon du Sel for providing the aromas. Correspondence should be addressed to L. Kaiser, Laboratoire de Neurobiologie Comparée des Invertébrés, INRA-CNRS (URA 1190), BP 23, la Guyonnerie, 91440 Bures-sur-Yvette, France (e-mail: kaiser@jouy.inra.fr).

-Accepted by previous editor, Vincent M. LoLordo et al. (Hymenoptera: Eucoilidae). Its major and preferred host is Drosophila melanogaster, but it can also develop in $D$. simulans, which is a less suitable host (Carton, Chibani, Haouas, \& Marrakachi, 1987). Both Drosophila species develop exclusively in fermenting fruits. In comparison with the generalist $L$. heterotoma, $L$. boulardi is therefore both host and host-habitat specialist. There is still discussion of the importance of learning in specialist insects. Different authors suggest that learning in generalists is more important than in specialists (see Vet et al., 1990, for review), and recent work indicates that there are differences between generalists and specialists in this respect (Poolman-Simons, Suverkropp, Vet, \& De Moed, 1992).

In a previous study (De Jong \& Kaiser, 1991), we demonstrated that $L$. boulardi can learn to respond to an odor through associative learning. However, the effects of learning were only tested in situations where no alternative odor was present, which gave no indication about the influence of learning on odor preference. In this paper, we report consequences of odor learning in a situation where this parasitoid has a choice between the learned odor and an alternative one. We also consider the influence of odor concentration, which is an important factor in odor learning by the honeybee (see, e.g., Marfaing, Rouault, \& Laffort, 1989; Pham-Delègue et al., 1993; Vareschi, 1971).

\section{GENERAL METHOD}

\section{Parasitoids}

L. boulardi (G464 strain), kindly provided by Y. Carton ${ }^{1}$, originated from Drosophila melanogaster collected from prickly pears (Opuntia ficus indica [L.]) in Tunisia (Nasrallah) in 1986. It was reared on Drosophila (mutant Rosie 295 strain) on an axenic medium. Pupae of parasitized Drosophila were collected, washed 
in $5 \%$ bleach, and rinsed in water before storage in tubes containing agar-agar and some honey, at $25^{\circ} \mathrm{C}$ and on a 12:12-h light:dark (LD) cycle. Thus the insects were not exposed to odors from their development medium during pupal and adult stages. After emergence of the parasitoids, these tubes were stored at $12^{\circ}-18^{\circ} \mathrm{C}(\mathrm{LD}$ 12:12) until needed.

\section{Odor Sources}

Glass capillaries $1 \mathrm{~cm}$ long and $1.56 \mathrm{~mm}$ in internal diameter, with one extremity sealed with wax and filled with banana or strawberry extract, served as odor sources in both conditioning procedure and behavioral testing. ${ }^{2}$ As each capillary released the same amount of aroma, variations in concentrations were obtained by changing the number of capillaries (see Experiments). We always used identical concentrations during conditioning and testing.

\section{Conditioning Procedure}

Five- to 7-day-old females were placed at $25^{\circ} \mathrm{C}$ (LD 12:12) and divided into five groups which were exposed to different conditioning procedures. Each group had a 1-h oviposition experience combined with a particular olfactory experience. For the oviposition experience, females were kept in a humidified airflow $(200 \mathrm{ml} / \mathrm{min}$ ) with agar-agar infested with $300-500$ first and second instar host larvae. In these conditions, a female performed about $50-60$ ovipositions. For one group, the airflow was not odorized. This naive group was labeled $\mathrm{N}+$, which indicates that the oviposition experience $(+)$ occurred in an odorless airflow $(\mathrm{N})$. Two other groups were subjected to associative conditioning to banana and strawberry odors, respectively. The I-h oviposition experience occurred in an airflow scented with the conditioning aroma. These groups were labeled $\mathrm{B}+$ and $\mathrm{S}+$, respectively. In addition to the naive group, two groups in which the 1 -h odor exposure to banana and strawberry respectively occurred $15-20 \mathrm{~min}$ after the $1-h$ oviposition experience served as controls (groups $\mathrm{N}+\mathrm{B}-$ and $\mathrm{N}+\mathrm{S}-$ ), because the odor exposure was not paired with the oviposition experience. All insects were stored at $25^{\circ} \mathrm{C}$ and LD 12:12 until needed for behavioral testing.

\section{Behavioral Testing}

Twenty-four hours after their conditioning, the insects were tested individually in a four-armed olfactometer $\left(T=25^{\circ} \mathrm{C}\right.$, light intensity: 850 lux) designed by Petterson (1970) and similar to the one used by Vet, Van Lenteren, Heymans, and Meelis (1983), with some modifications (De Jong \& Kaiser, 1991; see Figure 1 for a diagram of the device). One field was odorized with banana, an adjacent one with strawberry, and the others were not odorized (blank).

The airflow from each arm was adjusted to $200 \mathrm{ml} / \mathrm{min}$. The insect was introduced into a vertical Perspex entry tube (14 mm long and $10 \mathrm{~mm}$ in diameter), which was closed for the insect by a piece of gauze and connected to the silicon extraction tube. The observation started when the female entered the exposure chamber; it lasted $1 \mathrm{~min}$. Parasitoids needing more than $5 \mathrm{~min}$ to enter the chamber were discarded. We recorded the time spent in each scented field and the total time spent in the blank fields. Since the observation lasted $60 \mathrm{sec}$, random time allocation corresponded to a mean time of $15 \mathrm{sec}$ spent per field. After approximately $25 \mathrm{ob}-$ servations, the positions of the odorized fields in the exposure chamber were changed by odorizing other arms. Insects from the different groups of an experiment were tested in equal numbers on the same days (sizes of the groups are reported in the figures).

\section{Data Analyses}

Within a group, the Friedman analysis of variance based on ranks (one $d f$ ) was used to compare the times spent in banana and in strawberry odor. Across groups, we compared the magnitude of the preference between the scents, which we estimated from the difference between the time spent in banana and the time spent in



Figure 1. Diagrammatic top view of the olfactometer (adapted from Vet, Van Lenteren, Heymans, \& Meelis, 1983). Equal airflows arriving at each corner of the star-shaped exposure chamber are drawn through the chamber by a vacuum pump connected to the central hole of the device (incoming air is represented by the arrows). Each corner is connected to a system for air arrival including a glass vial to place an eventual odor source, a glass vial with water to humidify the air, and a flowmeter. In the exposure chamber, the dynamic of the airflow together with the shape of the chamber result in the creation of four well-delimited and adjacent flow fields of equal surface, which gather in the central hole. The insect is introduced in the center (see text for explanation) and then can perceive the four flow fields before entering the exposure chamber. In our experiments, banana (dashed area) and strawberry odor (spotted area) were delivered in two adjacent fields; the two other fields were not scented.

strawberry odor; the Mann-Whitney $U$ test or the Kruskal-Wallis analysis of variance based on ranks was used for this betweengroups comparison.

\section{EXPERIMENTS}

\section{Experiment 1}

Two capillaries filled with either banana or strawberry extract served as odor sources in this experiment. These odor concentrations were labeled B and S, respectively. Times spent in the odorized fields by the five groups described above are given in Figure 2. Friedman analysis on times spent in $\mathrm{B}$ and $\mathrm{S}$ revealed that $\mathrm{N}+$ females (which had oviposited but never experienced any fruit odor) were not attracted to $\mathrm{B}$ or $\mathrm{S}$. This was also true for the $\mathrm{N}+\mathrm{B}-$ group, in which oviposition experience and exposure to $\mathrm{B}$ were unpaired. A significant preference for $\mathrm{S}$ was observed in the $\mathrm{N}+\mathrm{S}-$ group (unpaired oviposition experience and exposure to $S$ ), in which females spent $45 \%$ of the time in S and $20 \%$ in B (Friedman $p<.05$ ). Associative conditioning induced a preference for the experienced odor, since $\mathrm{B}+$ females preferred B $(60 \%$ in B vs. $15 \%$ in S; Friedman $p<.001)$ and $\mathrm{S}+$ females spent almost all the time in $\mathrm{S}(92 \%$ in $\mathrm{S}$ vs. $3 \%$ in $\mathrm{B}$, Friedman $p<.001)$. The preference for $\mathrm{S}$ after associative conditioning ( $\mathrm{S}+$ ) was higher than after 


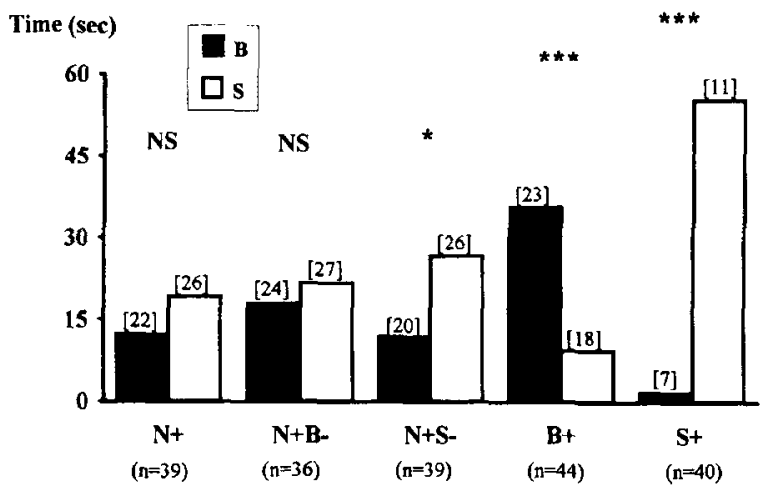

Figure 2. Mean time $[S D]$ spent in the fields scented with banana (in black) and strawberry aromas (in white) by differently experienced groups of female $\boldsymbol{L}$. boulardi: $\mathbf{N}+, \mathbf{N}+\mathbf{B}-, \mathbf{N}+\mathbf{S}-, \mathbf{B}+, \mathbf{S}+$ (see text for explanation). In this first experiment, two capillaries filled with banana (B) or strawberry (S) extract were used for conditioning and testing. The size of the groups, $n$, is indicated in parentheses. Within-group significant differences between the time spent in banana and in strawberry are indicated by asterisks (Friedman, ${ }^{\star} p<.05 ;{ }^{* *} p<.01 ;{ }^{* * *} p<.001 ;$ n.s., $p>.05$ ).

unpaired odor exposure $(\mathrm{N}+\mathrm{S}-)$ (Mann-Whitney, $p<$ $.05)$.

In order to test whether the observed preference for $S$ after exposure to $\mathrm{S}$ in the $\mathrm{N}+\mathrm{S}-$ group might reflect backward excitatory conditioning, we varied the time between the oviposition experience and the odor exposure, using intervals of 2 and $5 \mathrm{~h}$ in two additional groups. All insects were tested $24 \mathrm{~h}$ after their conditioning. The results presented in Table 1 show that comparable preferences for $S$ were induced with the original 15-min, the $2-\mathrm{h}$, and the 5-h intervals between oviposition and exposure to $S$. The three groups spent $45 \%-55 \%$ of the time in $S$ versus $10 \%-20 \%$ in $B$, and the magnitude of preference between $\mathrm{S}$ and $\mathrm{B}$ was not significantly different among these groups (Kruskal-Wallis $p>.05$ ).

\section{Experiment 2}

The perceived intensities of the odors may have influenced learning processes and preferences in behavioral testing (Marfaing et al., 1989; Pham-Delègue et al.,

Table 1

Mean Time (in Seconds, With Standard Deviations) Spent by Females Exposed to Strawberry (S) Odor After Their Oviposition Experience $(\mathbf{N}+\mathbf{S}-$ ) in the Fields Scented With Banana (B) and $S$, as a Function of Time Elapsed Between Oviposition Experience and Odor Exposure

\begin{tabular}{|c|c|c|c|c|c|c|}
\hline \multirow[b]{3}{*}{ Group } & \multirow[b]{3}{*}{$n$} & \multicolumn{4}{|c|}{ Time } & \multirow{3}{*}{$\begin{array}{l}\text { Friedman } \\
\chi^{2}, \text { B vs. S }\end{array}$} \\
\hline & & \multicolumn{2}{|c|}{ In $B$} & \multicolumn{2}{|c|}{ In $S$} & \\
\hline & & $M$ & $S D$ & $M$ & $S D$ & \\
\hline $\mathrm{N}+\mathrm{S}-{ }_{15-2}$ & 39 & 12 & 20 & 27 & 26 & $4.00, p<.05$ \\
\hline $\mathrm{N}+\mathrm{S}-2 \mathrm{~h}$ & 48 & 9 & 20 & 33 & 27 & $16.03, p<.001$ \\
\hline $\mathrm{N}+\mathrm{S}-5 \mathrm{~h}$ & 48 & 6 & 17 & 28 & 28 & $14.23, p<.001$ \\
\hline
\end{tabular}

Note-Elapsed time was $15-20 \mathrm{~min}, 2 \mathrm{~h}$, and $5 \mathrm{~h}$ for the three groups respectively. The initial concentrations $\mathrm{B} / \mathrm{S}$ were used for conditioning and testing.
1993). The fact that $S$ was learned better than $B$, as well as the effect of exposure to $S$ on subsequent behavior, could have been affected by the absolute and relative concentrations of both odors, whereby S dominated B. To obtain a better insight into this matter, we conducted experiments with the conditioning and testing of wasps in different odor concentrations. As in the first experiment, behavioral testing was done with the same odor concentrations as those used in the conditioning procedure. For this second experiment, the concentration of strawberry odor was decreased by half $(0.5 \mathrm{~S})$ while that of banana odor was kept in its original concentration (B). Insects were conditioned according to the same procedures as in the first experiment and groups were labeled $\mathrm{N}+, \mathrm{N}+\mathrm{B}-, \mathrm{N}+0.5 \mathrm{~S}-, \mathrm{B}+$, and $0.5 \mathrm{~S}+$.

As in the first experiment, oviposition experience alone $(\mathrm{N}+)$ and unpaired oviposition experience and exposure to $\mathrm{B}(\mathrm{N}+\mathrm{B}-)$ did not induce a significant preference for either odor (Figure 3). Interestingly, a significant preference for $\mathrm{B}$ over $0.5 \mathrm{~S}$ occurred in the $\mathrm{N}+0.5 \mathrm{~S}$ - group $(45 \%$ in $\mathrm{B}$ vs. $18 \%$ in $\mathrm{S}, p<.01)$, whereas the same treatment with the original concentration of $\mathrm{S}(\mathrm{N}+\mathrm{S}-)$ caused a preference for $S$ over $B$ in the first experiment (Figure 2). Associative conditioning in $\mathrm{B}+$ and $0.5 \mathrm{~S}+$ groups induced preferences for the experienced odors (Figure $3, \mathrm{~B}+: 70 \%$ in B vs. $7 \%$ in $0.5 \mathrm{~S}$, Friedman $p<$ $.001 ; 0.5 \mathrm{~S}+: 57 \%$ in $0.5 \mathrm{~S}$ vs. $27 \%$ in B, Friedman $p<$ $.01) . \mathrm{B}+$ females showed a stronger preference for $\mathrm{B}$ when $B$ was offered in the olfactometer together with $0.5 \mathrm{~S}$ rather than with $\mathrm{S}$ (Figures 2 and 3, Mann-Whitney $p<.05) .0 .5 \mathrm{~S}+$ females had a lower preference for $0.5 \mathrm{~S}$ over B than $\mathrm{S}+$ females had for $\mathrm{S}$ over B (Figures 2 and 3 , Mann-Whitney $p<.001$ ).

\section{Experiment 3}

Another way to decrease the relative concentration of strawberry odor in comparison with banana odor was to increase the concentration of banana odor. So, in this experiment, it was made twice as strong (2B) and $S$ was kept in its original concentration (S). These concentrations served for the conditioning and testing of the insects. They were conditioned according to the same procedures as in the first and second experiments, and the groups were labeled $\mathrm{N}+, \mathrm{N}+2 \mathrm{~B}-, \mathrm{N}+\mathrm{S}-, 2 \mathrm{~B}+$, and $\mathrm{S}+$.

$\mathrm{N}+, \mathrm{N}+2 \mathrm{~B}-$, and $\mathrm{N}+\mathrm{S}-$ treatments did not induce any significant preference for $2 \mathrm{~B}$ or $\mathrm{S}$ (Figure 4 ), whereas the $\mathrm{N}+\mathrm{S}-$ treatment caused a preference for $\mathrm{S}$ over B in the first experiment. After associative conditioning, females had a strong preference for their experienced odor $(2 \mathrm{~B}+: 77 \%$ in $2 \mathrm{~B}$ vs. $7 \%$ in $\mathrm{S}$, Friedman $p<.001 ; \mathrm{S}+: 78 \%$ in $\mathrm{S}$ vs. $5 \%$ in $2 \mathrm{~B}$, Friedman $p<$ $.001) .2 B+$ insects had a stronger preference for $2 \mathrm{~B}$ over $\mathrm{S}$ than $\mathrm{B}+$ insects had for $\mathrm{B}$ over $\mathrm{S}$ (Figures 2 and 4 , Mann-Whitney $p<.01$ ). The $\mathrm{S}+$ groups responded equally under both $2 \mathrm{~B} / \mathrm{S}$ and $\mathrm{B} / \mathrm{S}$ conditions (Figures 2 and 4, Mann-Whitney $p>.05$ ).

Over all the experiments, the proportion of discarded individuals-females that did not enter the exposure 


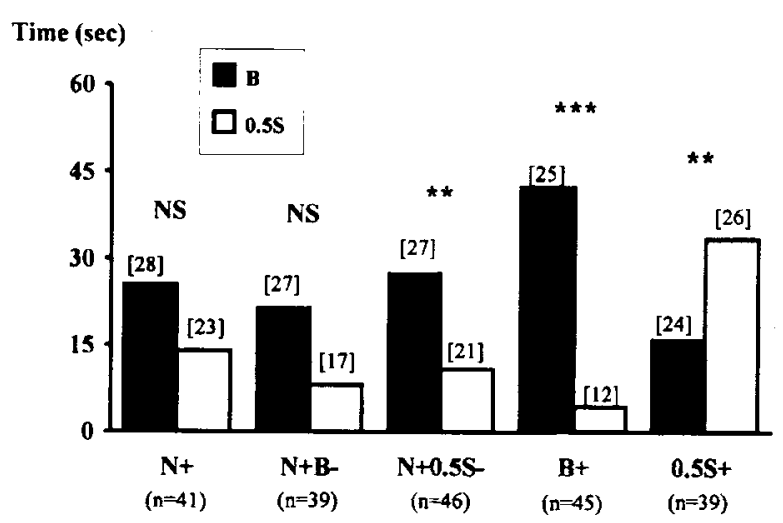

Figure 3. Results of the second experiment, in which the concentration ratio $\mathrm{B} / 0.5 \mathrm{~S}$ was used in the conditioning and testing procedure. See legend of Figure 1.

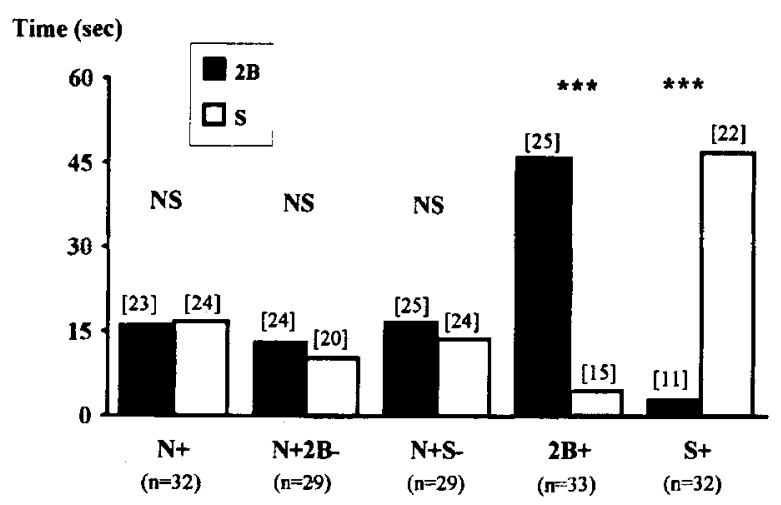

Figure 4. Results of the third experiment, in which the concentration ratio $2 \mathrm{~B} / \mathrm{S}$ was used in the conditioning and testing procedure. See legend of Figure 1.

chamber of the olfactometer within $5 \mathrm{~min}$-varied within groups between $5 \%$ and $20 \%$. The lowest values were generally observed after associative conditioning to banana or strawberry odor, with females being highly attracted to the odors.

\section{DISCUSSION}

Learning is generally considered to be an adaptive process through which animals can focus on stimuli that have been previously linked to a positive or negative experience. Induction of behavioral responses to odors that have been associated with oviposition, host products, or food is a common phenomenon in parasitic wasps.

This work shows that odor preferences depend on the olfactory experience of the wasps and on the absolute and relative concentrations of the odors. Odor concentrations do not affect associative learning in the same way as they affect nonassociative conditioning. After associative learning, the learned odor was preferred over the alternative one for all odor concentrations tested, but unpaired oviposition experience and exposure to straw- berry odor could induce a preference for either strawberry or banana odor, depending on the odor concentration (Figures 2-4). Exposure to strawberry odor, therefore, acts in a nonspecific way.

Initially, the strawberry odor was delivered $15-20 \mathrm{~min}$ after the 1-h oviposition experience to females of the $\mathrm{N}+\mathrm{S}-$ group. Induction of behavioral responses to a previously neutral stimulus after backward associative learning, in which the stimulus is presented shortly after a Pavlovian unconditioned stimulus, has been observed in rats (see, e.g., Keith-Lucas \& Guttman, 1975) and in landmark learning by honeybees (Couvillon, Leiato, \& Bitterman, 1991), but it was not found in odor learning by honeybees (Menzel, Greggers, \& Hammer, 1993). In our study, 2-h and 5-h intervals between oviposition and odor exposure resulted in the same significant preference for the experienced odor as did a 15-min interval (Table 1). This suggests that induced preference for strawberry odor did not arise from its backward association with the oviposition experience but rather was caused by nonassociative conditioning. Odor exposure is presumably the main component of the $\mathrm{N}+\mathrm{S}-$ treatment, since oviposition experience alone had no effect on odor response (group $\mathrm{N}+$ ) and exposure to strawberry after a delay as long as $5 \mathrm{~h}$ still elicited a preference for this odor. Increase in behavioral response to an olfactory stimulus after exposure to it has been rarely observed in insects thus far. As a matter of fact, it has not been found in bees (Menzel \& Bitterman, 1983; HuberBakchine \& Pham-Delègue, personal communication) or in flies (Nelson, 1971, cited in McGuire, 1984), but it has been reported in two specialist larval parasitic wasps exposed to host odors, Venturia canescens (Thorpe, 1938) and Cotesia rubecula (Kaiser \& Cardé, 1992). In these experiments, an increased response to the previously experienced scent was observed, whereas we also found an effect on the response to a nonexperienced scent. Since we only found an effect of odor exposure in the $\mathrm{N}+\mathrm{S}-$ groups and not in the $\mathrm{N}+\mathrm{B}-$ groups, occurrence of nonassociative conditioning appears to depend on the quality of the odor. In addition, the behavioral response induced by exposure to strawberry was determined by the concentration of the odors (Figures 2-4). Hence, behavioral changes resulting from exposure to an olfactory stimulus appear rather unpredictable, and since the responding to these stimuli was not reinforced, their adaptive significance is unclear.

The results show also that the concentration ratio between the odors offered in a choice situation can be extremely important in odor preference. $\mathrm{N}+\mathrm{S}-$ females exposed to the initial $\mathrm{S}$ concentration significantly preferred $S$ over $B$, but not over $2 B$ (Figures 2 and 4 ). Similarly, $\mathrm{B}+$ females showed a much stronger preference for $B$ over $0.5 \mathrm{~S}$ than over the initial concentration S (Figures 2 and 3 ). These findings emphasize the importance of choice possibilities and odor concentrations in the interpretation of odor preferences induced by olfactory conditioning. We used fruit odors, because we expected them to be learned by parasitoids of frugivorous hosts. 
The use of known chemicals will help us understand the influence of their qualitative and quantitative properties on odor learning.

L. boulardi is known to locate host-infested fruits by responding to volatiles produced by alcoholic fermentation due to development of yeast (Carton, 1978). This species parasitizes $D$. melanogaster in various fermenting fruits such as prickly pears, bananas (Carton, Boulétreau, Van Alphen, \& Van Lenteren, 1986), citrus (Hertlein, 1986), and moranda (Rubiaceae; Carton, personal communication). Females will lay eggs as long as suitable host larvae are available on a substrate (Carton et al., 1986). However, egg laying can be interrupted when host larvae become scarce (Carton et al., 1987; Janssen, 1989, for other species of drosophilid parasitoids) and parasitoids then have to search for another infested fruit. Our work suggests that experience of an infested fruit probably influences odor preference by females during their foraging behavior, as has been demonstrated for $L$. heterotoma in field conditions (Papaj \& Vet, 1990). In addition, we have shown that odor preference depends on factors other than associative learning, such as unrewarded odor exposure and absolute and relative concentrations of the perceived odors.

\section{REFERENCES}

CARTON, Y. (1978). Olfactory responses of Cothonaspis sp. (Hymenoptera: Cynipidae) to the food habit of its host (Drosophila melanogaster). Drosophila Information Service, 53, 183-184.

Carton, Y., Boulétreau, M., Van alphen, J. J. M., \& Van LENTEREN, J. C. (1986). The Drosophila parasitic wasps. In M. Ashburner, H. L. Carson, \& J. N. Thompson (Eds.), The genetics and biology of Drosophila (pp. 347-386). London: Academic Press.

Carton, Y., Chibani, F., Haouas, S., \& Marrakachi, M. (1987). Egg-laying strategy under natural conditions of Leptopilina boulardi, a hymenopteran parasitoid of Drosophila spp. Entomologia Experimentalis et Applicata, 43, 193-201.

Couvillon, P. A., Leiato, T., \& Bitterman, M. E. (1991). Learning by honeybees (Apis mellifera) on arrival at and departure from a feeding place. Journal of Comparative Psychology, 105, 177-184.

DE JONG, R., \& KAISER, L. (1991). Odour learning by Leptopilina boulardi, a specialist parasitoid. Journal of Insect Behavior, 4, 743 750.

DudaI, Y. (1989). Paradigms and research tools. In Y. Dudai (Ed.), The neurobiology of memory (pp. 19-34). New York: Oxford University Press.

Hertlern, M. B. (1986). Seasonal development of Leptopilina boulardi (Hymenoptera: Eucoilidae) and its hosts, Drosophila melanogaster and D. simulans (Diptera: Drosophilidae), in California. Environmental Entomology, 15, 859-866.

J ANSSEN, A. (1989). Optimal host selection by Drosophila parasitoids in the field. Functional Ecology, 3, 469-479.

KAISER, L., \& CARDÉ, R. T. (1992). In-flight orientation to volatiles from the plant-host complex in Cotesia rubecula (Hymenoptera: Braconidae): Increased sensitivity through olfactory experience. Physiological Entomology, 17, 62-67.
Keith-Lucas, T., \& Guttman, N. (1975). Robust-single-trial delayed backward conditioning. Journal of Comparative \& Physiological Psychology, 88, 468-476.

Lewis, W. J., \& TumLinson, J. H. (1988). Host detection by chemically mediated associative learning in a parasitic wasp. Nature, 331, 257259.

Marfaing, P., Rovault, J., \& Laffort, P. (1989). Effect of the concentration and nature of olfactory stimuli on the proboscis extension of conditioned honeybees Apis mellifica ligustica. Journal of Insect Physiology, 35, 949-955.

McGuire, T. R. (1984). Learning in three species of Diptera: The blowfly Phormia regina, the fruit fly Drosophila melanogaster and the house fly Musca domestica. Behavior Genetics, 14, 479-525.

Menzel, R., \& Bitterman, M. E. (1983). Learning in an unnatural situation. In F. Huber \& H. Markl (Eds.), Neuroethology and behavioural physiology (pp. 206-215). Berlin: Springer-Verlag.

Menzel, R., Greggers, U., \& Hammer, M. (1993). Functional organization of appetitive learning and memory in a generalist pollinator, the honey bee. In D. R. Papaj \& A. C. Lewis (Eds.), Insect learning (pp. 79-125). New York: Chapman \& Hall.

PAPAJ, D. R., \& VET, L. E. M. (1990). Odour learning and foraging success in the parasitoid, Leptopilina heterotoma. Journal of Chemical Ecology, 16, 3137-3150.

Petterson, J. (1970). An aphid sex attractant: I. Biological studies. Entomologica Scandinavica, 1, 63-73.

Pham-Deleggue, M. H., Ballez, O., Blight, M. M., Masson, C., Picard-Nizou, A. L., \& Wadhams, L. J. (1993). Behavioural discrimination of oilseed rape volatiles by the honeybee Apis mellifera L. Chemical Senses, 18, 483-494.

Poolman-Simons, M. T. T., Suverkropp, B. P., Vet, L. E. M., \& DE MoED, G. (1992). Comparison of learning in related generalist and specialist eucoilid parasitoids. Entomologia Experimentalis et Applicata, 64, 117-124.

THORPE, W. H. (1938). Further experiments on olfactory conditioning in a parasitic insect: The nature of the conditioning process. Proceedings of the Royal Society of London: Series B, 126, 370-397.

TurLings, T. E. C., Wäckers, F. L., Vet, L. E. M., LeWis, W. J., \& Tumlinson, J. H. (1993). Learning of host-finding cues by Hymenopterous parasitoids. In D. R. Papaj \& A. C. Lewis (Eds.), Insect learning (pp. 51-78). New York: Chapman \& Hall.

VARESCHI, E. (1971). Duftunterscheidung bei der Honigbiene: Einzelzell Ableitungen und Verhaltensreaktionen. Zeitschrift für vergleichende Physiologie, 75, 143-173.

VET, L. E. M., \& Groenewold, A. W. (1990). Semiochemicals and learning in parasitoids. Journal of Chemical Ecology, 16, 31193135.

Vet, L. E. M., Lewis, W. J., Papaj, D. R., \& Van Lenteren, J. C. (1990). A variable model for parasitoid foraging behavior. Journal of Insect Behavior, 3, 471-490.

Vet, L. E. M., Van Lenteren, J. C., Heymans, M., \& Meelis, E. (1983). An airflow olfactometer for measuring olfactory responses of hymenopterous parasitoids and other small insects. Physiological Entomology, 8, 97-106.

\section{NOTES}

1. Biologie et Génétique Evolutive, CNRS, F-91190, Gif-surYvette, France.

2. The aromas were kindly provided by Haarman \& Reimer S.A.R.L., 95310 Saint-Ouen-l'Aumône, France.

(Manuscript received November 25, 1991; revision accepted for publication May 18, 1994.) 